\title{
DEGENERATIVE JOINT DISEASES (OSTEOARTRTROSIS) AND QUALITY OF LIFE
}

\author{
V. Nikolova*, G. Prakova \\ Department of Internal Medicine and General Medicine, Faculty of Medicine, Trakia University, \\ Stara Zagora, Bulgaria
}

\begin{abstract}
Osteoarthritis (OA) is one of the most common chronic joint diseases and is the leading cause of permanent impairment in patients over 55 years of age. OA's social significance is also determined by their economic weight, which is between $1 \%$ and $2.5 \%$ of GDP in a number of Western European countries. The main subject of this work is to present the most common degenerative joint diseases and their impact on the quality of life. Material and Methods: Keywords and databases from published literature sources have been used in Scopus, Science Direct, Pub Web, Web of Science, In Cites, Access Medicine over the past 10 years. Results and discussion: OA affects most of the knee, hip and spinal joints, leads to disability and requires costly surgical treatment. The cost of care for OA patients is extremely high due to the need for frequent visits to a doctor, hospitalizations, rehabilitation procedures, expensive treatment and frequent complications resulting from the medication used. X-ray methods are a gold standard in OA diagnosis and the degree of degenerative changes using different scales and estimates depending on the location. OA aggravates quality of life, affecting total physical activity, selfesteem, mental and emotional state of the sick patients. The most commonly used method for assessing the quality of life of patients with osteoarthritis is a matter of SF-36v2, which is based on the norms assessed (NBS). It has been found that $80 \%$ of people with OA experience movement difficulties and $25 \%$ are unable to cope with their basic daily activities. Conclusion: Degenerative joint diseases are the most common diseases of the musculoskeletal system, which have a significant individual and social effect and significantly impair the quality of life of the patients.
\end{abstract}

Key words: osteoarthritis, quality of life

Osteoarthritis (OA) is one of the most common chronic joint diseases and is a leading cause of disability in patients over 55 years of age. The disease affects the articular cartilage and the subchondral bone that compromise the joint as a whole, most commonly causing damage to the joints / hip or knee. The illness rate in the adult population is $11 \%$ and $24 \%$, respectively, but can also affect the joints of the hands and fingers of the feet. OA suffers from approximately $80 \%$ of the earth's population aged 50 to 60 , but may appear earlier (3), more often affecting women.

Osteoarthritis develops as a result of joint degeneration-a process of progressive loss of articular cartilage accompanied by bone

\footnotetext{
*Correspondence to: Dr. Violeta Tsvetkova,

Department of Internal Diseases and General

Medicine, Faculty of Medicine, Thracian

University, Stara Zagora, e @ mail:

dr.cvetkova@abv.bg
}

remodeling, subchondral bone sclerosis and osteophyte formation. Age-related mechanical stress increases the risk of joint degeneration, but the exact pathophysiological mechanisms of the disease have not yet been fully elucidated. In English-language literature, the term osteoarthritis is spread as overlapping with osteoarthritis but distinct from rheumatoid arthritis with targeted structural damage to the hyaline cartilage (7).

Osteoarthritis, as a common high-frequency joint disease in middle and advanced age, has a high social significance and a high risk of disability. The classic symptoms of osteoarthritis are pain and impaired joint function. At the advanced stages of the disease, joint stiffness, muscular atrophy and limb deformities are observed. The main component of articular cartilage and synovial fluid is hyaluronic acid $(6,11)$, which is related to the visco-elastic properties of synovial fluid. In joint disease, this function and functional 
capacity are impaired (8). The most common are the bearing joints, especially knee joints, hips and joints of the spine. The negative impact of the disease on the overall condition and quality of life of patients is most pronounced when hip and knee joints are involved because pain and stiffness often result in disability requiring costly surgical treatment.Risk factors are overweight, hypodynamia, the presence of previous traumas or surgical interventions, bone deformities or concomitant diseases (diabetes mellitus, gout, etc.). It is important for the development of the disease to damage to cartilage cells (chondrocytes) that produce large a number of degrading enzymes and attack the major components of the cartilage matrix-collagen and proteoglycans. Hyaluronic acid content in synovial fluid decreases, viscosity changes with gradual destruction of the cartilage, reduction of joint mobility and joint fibrosis (9).

The target tissue of the injury is the hyaline cartilage, which consists of chondrocytes (5\%) and extracellular matrix (95\%). Chondrocytes produce an extracellular matrix containing a large amount of water (70\%), collagen fibrils (cartilage strength), proteoglycans (basic protein chain) and glycone amino glycine chondroitin sulfate, keratansulphate, proteoglycan aggregates / large number of proteoglycans attached to a hyaluronic acid molecule / (2). Proteoglycans and proteoglycan aggregates determine the elasticity of the hyaline cartilage. It lacks nerve and nerve endings, which determines the initial painless course of the disease, and the absence of blood and lymph vessels makes its trophic dependent on the other structures of the joint.

Osteoarthritis usually develops slowly and gradually worsens over time. Symptoms may range from mild to very severe and include motion pain (mechanical type), cracking or crunching sound in joint movement, stiffness and swelling, limited full motion range with loss of coordination, change in posture due to pain or stiffness. As the disease progresses, the subchondral bone is destroyed, inflammation develops, characterized by edema, warming, pain and restricted joint movements. Inflammation engages the synovial membrane and the capsule as well as the peristalsis / muscles, tendons, ligaments, burses / which are richly innervated and determine the occurrence of varying pain syndrome [1].Women develop osteoarthritis with a higher probability of having a higher risk of a more severe course of the disease (4). Hormonal factors are influenced, but the results of a number of studies do not prove a convincing relationship in this direction. Unbalanced weight and obesity are an unconditional risk factor for knee joints. Data from a number of studies have shown a reduction in the risk of symptomatic knee osteoarthritis in reducing body weight $(3,4)$.

Osteoarthritis affects people from all ethnic groups in all geographic areas of the world. According to WHO data, about $10 \%$ of people over 60 have osteoarthritis. Radiological evidence for osteoarthritis is present in more than half of the population over 65 and in $85 \%$ of those over the age of 70 . Between 2000 and 2010, the number of OA patients in the United States increased from 21 to 27 million, with the probable cause is aging and obesity. After the age of 40 , the incidence of OA has increased rapidly with each decade passed in all joints. (19) The annual economic weight of OA amounts to $1-2.5 \%$ of GDP in Western countries $(18,20)$ The cost of caring for patients with $\mathrm{OA}$ is extremely high due to the need for frequent visits to a doctor, hospitalization (mainly because of surgical interventions), costly medications, and dealing with complications due to NSAIDs.

The two most debilitating localizations of osteoarthritis are gonarthrosis and coxarthrosis (2). Gonarthrosis is the most common localization of osteoarthritis. Pain in the knee joint is mechanical, increases in load and decreases at rest, often influenced by weather / meteopathy / and in advanced stages and constant. Often there is stiffness and spasm of the obstructed musculature, and joint edema occurs when osteoarthritis is in the activation phase. Active gonarthrosis includes swelling, pain, warming, and severely restricted knee joint movements (17). In the advanced stages the knee is deformed genu varum type, the patient has a broken gait requiring the use of helper. There is a deterioration in quality of life, disability and endoprosthesis.

Coxarthrosis - the most severe localization of osteoarthritis. Here again, mechanical pain is a leading symptom. Initial damage to the cartilage is almost asymptomatic. Gradually, the patient becomes obstructed when climbing and climbing stairs while moving on uneven terrain. Joint swelling can not be demonstrated in joint status, unlike swelling in gonarthrosis. In the advanced stages there is hypothrophyte of the femoral musculature, shortening of the limb, disability. The quality of life is severely impaired and the only outcome is endoprosthesis. 
NIKOLOVA V. et al.

Osteoarthritis is a disease that degrades the quality of life, affecting the full physical activity, self-esteem and emotional balance of patients. $80 \%$ of people with OA experience movement difficulties and $25 \%$ are unable to cope with their basic daily activities $(3,4)$. For SF-Healh Surwey, the SF (Healh Surwey) is the most easily applicable and at the same time sufficiently informative with a high degree of reliability (0.90) (24). Quality Meric's $\mathrm{sF}$ health Survey - SF-36; SF-12; SF-8-healthcare is a scientifically valid and reliable measure of physical and mental health status. (25) The questionnaire is conducted for several minutes, and the responses received can be easily analyzed to measure and improve health. Translations are available in more than 170 languages and countries. In the past 20 years, over 19,000 studies have been conducted, creating a prerequisite for building an average score or a measure of the quality of life of patients with OA (26).

To investigate the onset and development of pain and physical functional impairment, the Western Ontario and McMaster Schools of Osteoarthritis Index (WOMAC) and their association with osteoarthritis on a large scale of the population are used. The index has painful and functional subscales, and is available in 65 alternative language formulas, validated in a 5 point phone and computer test version (28).

For the diagnosis of coxarthrosis and gonarthrosis, the $\mathrm{x}$-ray is gold.For the diagnosis of coxarthrosis and gonarthrosis, $\mathrm{X}$ ray is a gold standard that also determines the stage of the disease (24). The main radiographic features are narrowing of the joint, sclerosis in the subchondral bone, osteophytes, cysts in the underlying bone to deformities, subluxations and free bodies in the joints, as well as complete deletion of the joint gap. Often there is a mismatch between symptoms and radiographic changes. The rheumatological world community is developing Kallgren-Lawrence's osteoarthritis: Stage 1 - OA scarring, Stage 2 minor osteophytes and a slight narrowing of the joint gap, Stage 3 with pronounced osteophytes, advanced stenosis and subchondral sclerosis and Stage 4-manifold osteophytes, almost deleted joint, deformity, severely expressed subchondral sclerosis $(15,18)$. Other X-ray methods evaluate OA by direct bone distance measurement using MRI to visualize multiple articular structures. This method is especially useful when determining the effect of the use of drugs with a potentially modifying effect.16 The most widely used diagnostic criteria for
OA of knee and hip joints are developed by the American College of Rheumatology / 23). ACR criteria are more useful for refining OA on the knee than other localizations than for diagnosing it on its own in routine clinical practice. There is currently no grid-line that has been developed for the clinical diagnosis of knee joint OA. A broad application finds the evidence-based recommendations of the European League of Rheumatism (EULAR). They focus on the risk factors, symptoms, scars, and studies that can contribute to the clinical diagnosis. (23) Rare diagnosis and timely treatment of osteoarthritis is essential to slow down the progression of the disease. Contemporary treatment aims to relieve pain and stiffness, to control concomitant inflammation, improve joint function, and the quality of life of the patients, to slow the progression of the degenerative process in articular cartilage. Achieving the therapeutic goals is based on training the patient to reduce the load in injured joints, using reliefs, symptomatic treatment with painless NSAIDs, $\mathrm{CF}$, muscle relaxants, sedatives, correction of disturbed balance between breakdown and joint cartilage.Improving the quality of life of OA patients is of particular importance in reducing pain and functional impairment, reducing the daily dose of NSAIDs, slowing the progression of the disease and disabling the patients.

Hyaluronic acid injection may improve the synthesis of endogenous HA and proteoglycans from chondrocytes, prevent cartilage degradation and stimulate its regeneration. In addition, HA can reduce the production of substances, the effects of inflammation and degrading enzymes, and reduce pain impulses (2). Hondroprotection is the modern concept in the field of rheumatology and orthopedics, which is constantly developing and aims to build and protect the joint cartilage, to slow down its wasting. Chondroprotectors are used for both prevention and treatment of OA disease (20). The main proven chondroprotectors are glucosamine sulphate, chondroitin sulfate, lubricants (hyaluronic acid), collagen products. Physiotherapeutic and rehabilitation procedures are not irrelevant at all stages of the disease. The endoprosthesis of the affected joints in the 4th stage restores the patient's motor capacity and restores its quality of life (19).

Conclusion: Osteoarthritis is a widespread and debilitating disease of the musculoskeletal system, resulting in substantial individual and public expenditure. The most frequent are the 
degenerative changes of the hip, knee joints and the spine. Osteoarthritis is a disease that degrades the quality of life of the patients and leads to significant costs in healthcare. To reduce these adverse health effects, it is important to have early diagnosis and adequate treatment of the disease, appropriate rehabilitation and training of the patients.

\section{REFERENCES}

1. Kolarov Zl. Rheumatic pain MLN Ltd., Sofia 2000

2. Kolarov Zl. The series of the twelve most common rheumatic diseases. Sofia: KLMN, 2011.

3. Nestrova R. Arthrosis is treatable 1 part STING.11 / 2004,38-39.

4. Nestorova R. Arthrosis Disease - the Most Common Disease in Everyday Practice 2011, Vol. Medison INSS 1314-0345; 4,7175 ;

5. Nestorova P. Osteoarthritis - the most common joint disease; MedikArt; 6 / 2011,30-33;

6. Peicheva, B. Comparative studies on synovial fluid in inflammatory and degenerative joint diseases. Dis. C.1987.

7. Rashkov R, Y.Sheitanov Practical Guide to Rheumatology, CIM 2002

8. Sheytanov Y. Osteoarthrosis, Medicine and Physical Education, 1990

9. Sheytanov Y, I.Sheytanov, Osteoarthrosis, 2006

10.Sheytanov, J. Rheumatic diseases - short course. C., CIM, 1998.

11.Goldberg, D., B. Amor and A. Chevrous. Rheumatology (From Anatomy to Diagnosis), C. Med. An.

12.Chandrate P, Roddy E, Clarson L, et al.Health related quality of life, and systematic review.Reumatology (Oxf).2013; 52 (11), 2013-2040.

13.Cross $M$ The global burden of hip and knee osteoarthritis from diobal burden of disease 2010 studyqAnn Reum Dis 2014; 73; 1323 1330

14.Gydek A., et al.Przegl Lek.2011; 68 [6]; 307-10.

15.Kelley, W. N. et al., The Textual of Reumatology, 4th Edition, Philadelphia, W.B.Saunders comp., 1993.
16.Klippel,J.H.etP.A.Dieppe.Rheumatology.L ondon, Mosby, 1994.

17.Koopman, W.Arhritis and Allied Conditions. A Texbook of Rheumatology.13th by Ed.Baltimore, Williams \& Wilkins.1997.

18.McCarty, D.J.Arthritis and Allied Condicions. Philadelphia-London, Lea \& Febiger, 1993.

19.M.Lotz.Osteoarthritis, year 2011 in review: biology.Osteoarthritis and Cartilage 20 [2012] 192-196

20.Moreland LW.Intra-articular hyaluronan [hyaluronic acid] and hylans for the treatment of osteoarthritis: mehanisms of action.Arthritis Res Ther.2003; 5 [12] 5467; Epub 2003 jan14, Rewiewd Fisc., 1994.

21.Petrella RJ et al., Reumatol.2006 May; 33 [5]; 951-6.

22.Reuckeweart, A.Maladies des os artic articulations. Paris, Flammarion, 1989.

23.Welger, S. T., B. L. Belsa and E. P. Galll, Clinical Care in the Reumatic Diseases, Atlanta, American College of Rheumatology, 1996.

24.Ware, J., Cathy, D. et al. , 1994; Vileikyte, L., Peyrot, M .; et al.2003

25.Were, J.SF-36 Health Survey Update Spine 2000. 3130-3139.

26.www.sf-8.org

27.(1992). Double-blind randomized controlled trials of sodium meclofenate (Meclomen) and diclofenac sodium (Voltaren): post validation reapplication of the WOMAC Osteoarthritis index. Journal of Rheumatology, 19: 153-9.

28.Bellamy N., Buchanan, W.W., Goldsmith, C.H., Campbell, J., \& Stitt, L.W. (1988). Validation study of WOMAC: a health status tool for measuring clinically important patient relevant outcomes to antirheumatic drug therapy in patients with osteoarthritis of the hip or knee. Journal of Rheumatology, 15: 1833-40.

29.Bellamy, N. (2005). The WOMAC Knee and Hip Osteoarthritis Indices: Development, validation, globalization and influence on the development of AUSCAN Hand Osteoarthritis Indices. Clinical and Experimental Rheumatology; 23 (Suppl. 39); 148-153 